\title{
Stability Analysis and Stabilization for Discrete-Time Fuzzy Systems With Time-Varying Delay
}

\author{
Huijun Gao, Member, IEEE, Xiuming Liu, and James Lam, Senior Member, IEEE
}

\begin{abstract}
This paper is concerned with the problems of stability analysis and stabilization for discrete-time Takagi-Sugeno fuzzy systems with time-varying state delay. By constructing a new fuzzy Lyapunov function and by making use of novel techniques, an improved delay-dependent stability condition is obtained, which is dependent on the lower and upper delay bounds. The merit of the proposed stability condition lies in its reduced conservatism, which is achieved by avoiding the utilization of some bounding inequalities for the cross products between two vectors. Then, a delay-dependent stabilization approach based on a parallel distributed compensation scheme is developed for both state feedback and observer-based output feedback cases. The proposed stability and stabilization conditions are formulated in terms of linear matrix inequalities, which can be solved efficiently by using existing optimization techniques. Two illustrative examples are provided to demonstrate the effectiveness of the results proposed in this paper.
\end{abstract}

Index Terms-Delay dependence, fuzzy systems, linear matrix inequality (LMI), stabilization, time-delay systems.

\section{INTRODUCTION}

$\mathbf{S}$ INCE fuzzy sets were proposed by Zadeh [35], fuzzy logic control has developed into a conspicuous and successful branch of automation and control theory. In many model-based fuzzy control approaches, the well-known Takagi-Sugeno (T-S) fuzzy model [26] has attracted much attention. The common practice based on T-S fuzzy model is as follows. The T-S fuzzy model is employed to represent or approximate a nonlinear system, which is described by a family of fuzzy IF-THEN rules that represent local linear input-output relations of the system. The overall fuzzy model of the system is achieved by smoothly blending these local linear models together through membership functions. It has a convenient dynamic structure so that some well-established linear system theories can be easily applied for the theoretical analysis and design of nonlinear systems. Therefore, the last decade has witnessed a rapidly growing interest in T-S fuzzy systems, and

Manuscript received January 25, 2008; revised June 17, 2008 and June 18, 2008. First published December 9, 2008; current version published March 19, 2009. This work was supported in part by the National Natural Science Foundation of China (60504008 and 60825303), by the Research Fund for the Doctoral Program of Higher Education of China (20070213084), by the Fok Ying Tung Education Foundation (111064), and by HKU CRCG 200707176077. This paper was recommended by Associate Editor W. Dixon.

H. Gao and X. Liu are with the Department of Space Control and Inertial Technology Research Center, Harbin Institute of Technology, Harbin 150001, China (e-mail: hjgao@ hit.edu.cn; liuxiuming22@gmail.com).

J. Lam is with the Department of Mechanical Engineering, The University of Hong Kong, Hong Kong (e-mail: james.lam@hku.hk).

Digital Object Identifier 10.1109/TSMCB.2008.2003449 many important results have been reported. To mention a few, the problem of stability analysis is investigated in [5], [16], [22], and [30], and stabilizing and $H_{\infty}$ control designs are reported in [13], [15], [22], [23], [25], [28], [29], [32], and [36].

On the other hand, time delay exists commonly in many practical systems such as chemical processes and networked systems, which has been generally regarded as the main source of instability and poor performance. Therefore, considerable attention [3], [6], [7], [10], [24], [31] has been devoted to the problems of analysis and synthesis for time-delay systems. The existing results for stability analysis can be classified into two types: delay-independent results [3], [11], [21] and delay-dependent results [6], [7], [9], [20], [34]. The former is irrespective of the delay size, whereas the latter usually contains the delay information. Since time delay is not taken into consideration, delay-independent results are generally regarded as being more conservative than delay-dependent ones, particularly when the delay size is small. It is worth noting that most of the aforementioned results are for linear systems. However, there exist many complex nonlinear systems with time delay in practical situations, and thus, it is natural to investigate nonlinear systems with time delay via the corresponding $\mathrm{T}-\mathrm{S}$ fuzzy model [1].

In fact, based on recent progress in linear time-delay systems, a number of important analysis and synthesis results have been derived for T-S fuzzy systems with time delay [2], [12], [14], [17], [33], [37]. To mention a few, Lien [12] and Liu [17] studied continuous-time systems with time delay based on a (nonfuzzy) Lyapunov function, and discrete-time T-S fuzzy systems with time delay were investigated in [2], [33], and [37]. It is worth pointing out that to derive delay-dependent stability conditions, some model transformations were usually performed to the original system, and thus, an inequality was inevitably employed to bound the inner product between two vectors, which gave rise to possible conservatism. Another point worth mentioning is that most of the previous results [2], [33], [37] for discrete-time fuzzy delay systems assume that the time delay was constant. This assumption facilitates the treatment of the considered problems but has inevitably limited the applicability of the obtained results. The main reason is apparent since, in most practical situations, the delay is time varying. Furthermore, if the delay is constant, we can transform the delayed system into a delay-free one by using state augmentation techniques. In this way, stability of such systems can be readily tested by employing classical results on stability analysis. However, the state augmentation technique is usually not applicable to the time-varying delay case. The reason is that for time-varying delay systems, the transformed systems 
usually have time-varying matrix coefficients, which are apparently difficult to analyze using available tools. According to the best of the authors' knowledge, little progress has been reported for the stability analysis of discrete-time fuzzy systems with time-varying state delay, which motivates this paper.

In this paper, the problems of delay-dependent stability analysis and stabilization for discrete-time T-S fuzzy systems with time-varying state delay are studied. First, a new fuzzy Lyapunov function is constructed to derive a delaydependent stability condition for the open-loop fuzzy systems. No model transformation is involved in the derivation of the delay-dependent stability condition. The merit of the proposed condition lies in its reduced conservatism, which is achieved by circumventing the utilization of some bounding inequalities for the cross product between two vectors. Then, based on the parallel distributed compensation (PDC) scheme [8], the delay-dependent stabilization conditions are worked out for the closed-loop fuzzy systems. Both state feedback and observerbased output feedback control cases are considered. The proposed stability and stabilization conditions are represented in terms of linear matrix inequalities (LMIs), which can be solved efficiently by using existing optimization techniques.

This paper is organized as follows. Section II provides preliminaries and the formulation of discrete-time T-S fuzzy systems with time-varying state delay. Delay-dependent stability analysis is presented in Section III. In Section IV, delaydependent stabilization conditions are provided for both state feedback and observed-based output feedback control cases. Simulation results are given in Section $\mathrm{V}$ to illustrate the effectiveness of the proposed method. Finally, conclusion is drawn in Section VI.

Notation: The notation used throughout this paper is fairly standard. The superscript " $\mathrm{T}$ " stands for matrix transposition; $\mathbb{R}^{n}$ denotes the $n$-dimensional Euclidean space; the notation $P>0(\geq 0)$ means that $P$ is real symmetric and positive definite (semidefinite); and $\mathbb{R}^{m \times n}$ is the set of all real matrices of dimension $m \times n$. In symmetric block matrices or complex matrix expressions, we use an asterisk $(*)$ to represent a term that is induced by symmetry, and for a matrix $A, \operatorname{sym}(A)$ denotes $A+A^{\mathrm{T}}$, and $\operatorname{diag}\{\ldots\}$ stands for a block-diagonal matrix. Matrices, if their dimensions are not explicitly stated, are assumed to be compatible for algebraic operations.

\section{Problem Formulation}

Many nonlinear systems can be expressed as a set of linear systems in local operating regions. Consider a discrete nonlinear system with time-varying delay in the state, which is represented by the following T-S fuzzy model.

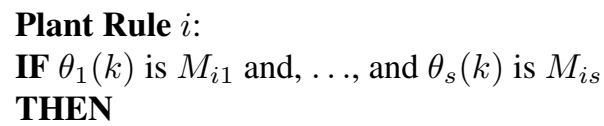

$$
\begin{aligned}
x(k+1) & =A_{i} x(k)+A_{d i} x(k-d(k))+B_{i} u(k) \\
y(k) & =C_{i} x(k)+C_{d i} x(k-d(k)) \\
x(k) & =\varphi(k), \quad k=-d_{M},-d_{M}+1, \ldots, 0 .
\end{aligned}
$$

Here, $i \in \Re=\{1,2, \ldots, r\}$, where $r$ is the number of IF-THEN rules; $M_{i j}$ is the fuzzy set; $x(k) \in \mathbb{R}^{n}$ is the state vector; $u(k) \in \mathbb{R}^{m}$ is the input vector; $y(k) \in \mathbb{R}^{q}$ is the measurement output vector; $x(k)=\varphi(k), k=-d_{M},-d_{M}+$ $1, \ldots, 0$, is a given initial condition sequence; $\theta(k)=$ $\left[\theta_{1}(k), \theta_{2}(k), \ldots, \theta_{s}(k)\right]$ are the premise variables; $A_{i}, A_{d i}$, $B_{i}, C_{i}$, and $C_{d i}$ are the constant matrices with appropriate dimensions; and $d(k)$ is a time-varying delay in the state. A natural assumption on $d(k)$ can be made as follows.

Assumption 1: The time delay $d(k)$ is assumed to be time varying and satisfy $0<d_{m} \leq d(k) \leq d_{M}$, where $d_{m}$ and $d_{M}$ are the constant positive scalars representing the lower and upper delay bounds, respectively.

Remark 1: The assumption on the time delay $d(k)$ in Assumption 1 characterizes the real situation in many practical applications. A typical example containing time delays can be found in networked control systems, where the delays induced by the network transmission are actually time varying, and can be assumed to have lower and upper delay bounds without loss of generality. It is worth noting that by assuming $d_{m}=d_{M}=d$, the time-varying delay $d(k)$ reduces to a constant delay $d$, which has been widely studied in the literature [2], [33], [37]. However, to the best of the authors' knowledge, few papers consider the time-varying delay case for discrete-time fuzzy systems.

It is assumed that the premise variables do not depend on the input variable $u(k)$, which is needed to avoid a complicated defuzzification process of fuzzy controllers [27]. Given a pair of $(x(k), u(k)))$, the outputs of the fuzzy system are inferred as

$$
\begin{aligned}
x(k+1) & =\sum_{i=1}^{r} h_{i}(\theta(k))\left\{A_{i} x(k)+A_{d i} x(k-d(k))+B_{i} u(k)\right\} \\
y(k) & =\sum_{i=1}^{r} h_{i}(\theta(k))\left\{C_{i} x(k)+C_{d i} x(k-d(k))\right\}
\end{aligned}
$$

where the fuzzy basis functions are given by

$$
h_{i}(\theta(k))=\frac{\mu_{i}(\theta(k))}{\sum_{i=1}^{r} \mu_{i}(\theta(k))}
$$

with $\mu_{i}(\theta(k))=\prod_{j=1}^{s} M_{i j}\left(\theta_{j}(k)\right)$, where $M_{i j}\left(\theta_{j}(k)\right)$ is the grade of membership of $\theta_{j}(k)$ in $M_{i j}$. In this paper, it is assumed that

$$
\mu_{i}(\theta(k)) \geq 0, i \in \Re, \quad \text { and } \quad \sum_{i=1}^{r} \mu_{i}(\theta(k))>0
$$

for all $k$ 's. Therefore, $\quad h_{i}(\theta(k)) \geq 0, \quad i \in \Re, \quad$ and $\sum_{i=1}^{r} h_{i}(\theta(k))=1$. Then, the discrete-time T-S fuzzy model with time-varying delay in the state is given by

$$
\begin{aligned}
x(k+1) & =\bar{A}(k) x(k)+\bar{A}_{d}(k) x(k-d(k))+\bar{B}(k) u(k) \\
y(k) & =\bar{C}(k) x(k)+\bar{C}_{d}(k) x(k-d(k))
\end{aligned}
$$


where

$$
\begin{array}{rlrl}
\bar{A}(k) & =\sum_{i=1}^{r} h_{i}(\theta(k)) A_{i} \quad \bar{A}_{d}(k)=\sum_{i=1}^{r} h_{i}(\theta(k)) A_{d i} \\
\bar{B}(k) & =\sum_{i=1}^{r} h_{i}(\theta(k)) B_{i} \quad \bar{C}(k)=\sum_{i=1}^{r} h_{i}(\theta(k)) C_{i} \\
\bar{C}_{d}(k) & =\sum_{i=1}^{r} h_{i}(\theta(k)) C_{d i} . &
\end{array}
$$

\section{Delay-Dependent Stability Analysis}

In this section, we will analyze the delay-dependent stability for fuzzy time-delay system. Without loss of generality, let $u(k)=0$ in the fuzzy time-delay system (3), which is given by

$$
x(k+1)=\bar{A}(k) x(k)+\bar{A}_{d}(k) x(k-d(k)) .
$$

Since the delay $d(k)$ is time varying, it is not possible to transform system (4) into a delay-free system by augmenting the state variables. The reason is that for time-varying delay systems, the transformed systems usually have time-varying matrix coefficients, which are apparently difficult to analyze using available tools. Furthermore, in the existing literature [2], [33], to bring the information of the delay size into the final result (to achieve delay dependence), some model transformations were performed to the original system in (4), and thus, inequalities were inevitably employed to bound the inner product between two vectors, which gave rise to possible conservatism. Here, our purpose is to first obtain an improved delay-dependent stability condition for system (4) with time-varying delay. For the convenience of notations, we use the fuzzy basis function in (2) to denote the following functions:

$$
\begin{aligned}
& \bar{P}(k)=\sum_{i=1}^{r} h_{i}(\theta(k)) P_{i} \\
& \bar{Q}(k)=\sum_{i=1}^{r} h_{i}(\theta(k)) Q_{i} \\
& \bar{Z}(k)=\sum_{i=1}^{r} h_{i}(\theta(k)) Z_{i}
\end{aligned}
$$

then we have the following theorem.

Theorem 1: The fuzzy time-delay system in (4) is asymptotically stable for time-varying delay $d(k)$ satisfying $0<d_{m} \leq$ $d(k) \leq d_{M}$, if there exist matrices $P_{i}>0, Q_{i}>0, Z_{i}>0$, and $S_{1 i}, S_{2 i}$, such that the following LMIs hold:

$$
\begin{aligned}
\Pi_{\text {stlii }}<0, & i, j, s, t, l \in \Re \\
\Pi_{s t l i j}+\Pi_{\text {stlji }}<0, & 1 \leq i<j \leq r
\end{aligned}
$$

where

$$
\begin{aligned}
\Pi_{s t l i j} & =\left[\begin{array}{ccc}
\Psi_{i s}+\operatorname{sym}\left(\Xi_{2 i}\right) & \sqrt{d_{M}} S_{i} & \Phi_{1, i j t} \\
* & -Z_{l} & 0 \\
* & * & \Phi_{2, j t}
\end{array}\right] \\
\Psi_{i s} & =\left[\begin{array}{cc}
-P_{i}+\tau Q_{i} & 0 \\
0 & -Q_{s}
\end{array}\right] \\
\Xi_{2 i} & =\left[\begin{array}{ll}
S_{i} & -S_{i}
\end{array}\right] \\
S_{i} & =\left[\begin{array}{cc}
S_{1 i} \\
S_{2 i}
\end{array}\right] \\
\Phi_{1, i j t} & =\left[\begin{array}{cc}
A_{i}^{\mathrm{T}} P_{t} & \sqrt{d_{M}}\left(A_{i}^{\mathrm{T}}-I\right) Z_{j} \\
A_{d i}^{\mathrm{T}} P_{t} & \sqrt{d_{M}} A_{d i}^{\mathrm{T}} Z_{j}
\end{array}\right] \\
\Phi_{2, j t} & =\left[\begin{array}{cc}
-P_{t} & 0 \\
0 & -Z_{j}
\end{array}\right] \\
\tau & =d_{M}-d_{m}+1 .
\end{aligned}
$$

Proof: Denote $\eta(k)=x(k+1)-x(k)$. Then, for the fuzzy time-varying delay system in (4), we have

$$
\begin{gathered}
\eta(k)=[\bar{A}(k)-I] x(k)+\bar{A}_{d}(k) x(k-d(k)) \\
x(k)-x(k-d(k))=\sum_{l=k-d(k)}^{k-1} \eta(l) .
\end{gathered}
$$

To prove the theorem, we choose the following fuzzy Lyapunov function:

$$
V(k)=V_{1}(k)+V_{2}(k)+V_{3}(k)+V_{4}(k)
$$

where

$$
\begin{aligned}
& V_{1}(k)=x^{\mathrm{T}}(k) \bar{P}(k) x(k) \\
& V_{2}(k)=\sum_{l=k-d(k)}^{k-1} x^{\mathrm{T}}(l) \bar{Q}(l) x(l) \\
& V_{3}(k)=\sum_{j=-d_{M+2}} \sum_{l=k+j-1}^{-d_{m}+1} x^{\mathrm{T}}(l) \bar{Q}(l) x(l) \\
& V_{4}(k)=\sum_{i=-d_{M}}^{-1} \sum_{l=k+i}^{k-1} \eta^{\mathrm{T}}(l) \bar{Z}(l) \eta(l)
\end{aligned}
$$

and $\bar{P}(k), \bar{Q}(l)$, and $\bar{Z}(l)$ are defined in (5) with $P_{i}, Q_{l}$, and $Z_{l}$ being the positive definite matrices to be determined. Define 
$\Delta V(k)=V(k+1)-V(k)$. Along the solution of system (4), we have

$$
\begin{aligned}
& \Delta V_{1}(k)=x^{\mathrm{T}}(k+1) \bar{P}(k+1) x(k+1)-x^{\mathrm{T}}(k) \bar{P}(k) x(k) \\
& \Delta V_{2}(k)=\sum_{l=k-d(k+1)+1}^{k} x^{\mathrm{T}}(l) \bar{Q}(l) x(l)-\sum_{l=k-d(k)}^{k-1} x^{\mathrm{T}}(l) \bar{Q}(l) x(l) \\
& =x^{\mathrm{T}}(k) \bar{Q}(k) x(k)-x^{\mathrm{T}}(k-d(k)) \\
& \times \bar{Q}(k-d(k)) x(k-d(k)) \\
& +\sum_{l=k-d(k+1)+1}^{k-1} x^{\mathrm{T}}(l) \bar{Q}(l) x(l) \\
& -\sum_{l=k-d(k)+1}^{k-1} x^{\mathrm{T}}(l) \bar{Q}(l) x(l) \leq x^{\mathrm{T}}(k) \bar{Q}(k) x(k) \\
& -x^{\mathrm{T}}(k-d(k)) \times \bar{Q}(k-d(k)) x(k-d(k)) \\
& +\sum_{l=k-d_{M}+1}^{k-d_{m}} x^{\mathrm{T}}(l) \bar{Q}(l) x(l) \\
& \Delta V_{3}(k)=\sum_{j=-d_{M}+2}^{-d_{m}+1}\left\{x^{\mathrm{T}}(k) \bar{Q}(k) x(k)-x^{\mathrm{T}}(k+j-1)\right. \\
& \times \bar{Q}(k+j-1) x(k+j-1)\} \\
& =\left(d_{M}-d_{m}\right) x^{\mathrm{T}}(k) \bar{Q}(k) x(k) \\
& -\sum_{l=k-d_{M}+1}^{k-d_{m}} x^{\mathrm{T}}(l) \bar{Q}(l) x(l) \\
& \Delta V_{4}(k)=\sum_{i=-d_{M}}^{-1}\left\{\eta^{\mathrm{T}}(k) \bar{Z}(k) \eta(k)-\eta^{\mathrm{T}}(k+i) \bar{Z}(k+i) \eta(k+i)\right\} \\
& =d_{M} \eta^{\mathrm{T}}(k) \bar{Z}(k) \eta(k)-\sum_{l=k-d_{M}}^{k-1} \eta^{\mathrm{T}}(l) \bar{Z}(l) \eta(l) \\
& \leq d_{M} \eta^{\mathrm{T}}(k) \bar{Z}(k) \eta(k)-\sum_{l=k-d(k)}^{k-1} \eta^{\mathrm{T}}(l) \bar{Z}(l) \eta(l) .
\end{aligned}
$$

Based on (11), for any matrices

$$
\bar{S}(k)=\left[\begin{array}{l}
\bar{S}_{1}(k) \\
\bar{S}_{2}(k)
\end{array}\right]
$$

where

$$
\bar{S}_{1}(k)=\sum_{i=1}^{r} h_{i}(\theta(k)) S_{1 i} \quad \bar{S}_{2}(k)=\sum_{i=1}^{r} h_{i}(\theta(k)) S_{2 i}
$$

we have

$$
\begin{aligned}
\Pi(k)=2 & \frac{1}{d(k)} \sum_{l=k-d(k)}^{k-1}\left[x^{\mathrm{T}}(k) \quad x^{\mathrm{T}}(k-d(k))\right] \\
& \times \bar{S}(k)[x(k)-d(k) \eta(l)-x(k-d(k))]=0 .
\end{aligned}
$$

Define $\xi(k, l)=\left[\begin{array}{lll}x^{\mathrm{T}}(k) & x^{\mathrm{T}}(k-d(k)) \quad \eta^{\mathrm{T}}(l)\end{array}\right]^{\mathrm{T}}$, and then, from (12)-(15), we have

$$
\begin{aligned}
\Delta V(k) \leq & {\left[\bar{A}(k) x(k)+\bar{A}_{d}(k) x(k-d(k))\right]^{\mathrm{T}} \bar{P}(k+1) } \\
& \times\left[\bar{A}(k) x(k)+\bar{A}_{d}(k) x(k-d(k))\right] \\
& +\left(d_{M}-d_{m}+1\right) x^{\mathrm{T}}(k) \bar{Q}(k) x(k) \\
& -x^{\mathrm{T}}(k-d(k)) \bar{Q}(k-d(k)) x(k-d(k)) \\
& +d_{M}\left\{[\bar{A}(k)-I] x(k)+\bar{A}_{d}(k) x(k-d(k))\right\}^{\mathrm{T}} \\
& \times \bar{Z}(k)\left\{[\bar{A}(k)-I] x(k)+\bar{A}_{d}(k) x(k-d(k))\right\} \\
& -x^{\mathrm{T}}(k) \bar{P}(k) x(k)-\sum_{l=k-d(k)}^{k-1} \eta^{\mathrm{T}}(l) \bar{Z}(l) \eta(l)+\Pi(k) \\
= & \frac{1}{d(k)} \sum_{l=k-d(k)}^{k-1} \xi^{\mathrm{T}}(k, l) \\
& \times\left\{\sum_{s=1}^{r} \sum_{t=1}^{r} \sum_{l=1}^{r} h_{s}(\theta(k-d(k)))\right. \\
& \times h_{t}(\theta(k+1)) h_{l}(\theta(k)) \\
& \times \sum_{i=1}^{r} h_{i}(\theta(k)) \Phi_{s t l i i}+\sum_{i=1}^{r-1} \sum_{j=i+1}^{r} h_{i}(\theta(k)) \\
& \left.\left.\times h_{j}(\theta(k))\left(\Phi_{s t l i j}+\Phi_{s t l j i}\right)\right]\right\} \xi(k, l)
\end{aligned}
$$

where the expressions $\Phi_{\text {stlij }}$ and $\Xi_{1, i j t}$ are shown at the bottom of the page, and $\Xi_{2 i}, \Psi_{i s}$, and $S_{i}$ are defined in (9). On the other hand, noticing $0<d_{m} \leq d(k) \leq d_{M}$, by Schur complement, from (6) and (7), it is not difficult to get

$$
\begin{aligned}
\Phi_{\text {stlii }}<0, & i, j, s, t, l \in \Re \\
\Phi_{\text {stlij }}+\Phi_{\text {stlji }}<0, & 1 \leq i<j \leq r .
\end{aligned}
$$

$$
\begin{aligned}
\Phi_{s t l i j} & =\left[\begin{array}{cc}
\Xi_{1, i j t}+\operatorname{sym}\left(\Xi_{2 i}\right)+\Psi_{i s} & -\sqrt{d(k)} S_{i} \\
* & -\bar{Z} l
\end{array}\right] \\
\Xi_{1, i j t} & =\left[\begin{array}{cc}
A_{i}^{\mathrm{T}} P_{t} A_{i}+d_{M}\left(A_{i}^{\mathrm{T}}-I\right) Z_{j}\left(A_{i}-I\right) & A_{i}^{\mathrm{T}} P_{t} A_{d i}+d_{M}\left(A_{i}^{\mathrm{T}}-I\right) Z_{j} A_{d i} \\
* & A_{d i}^{\mathrm{T}} P_{t} A_{d i}+d_{M} A_{d i}^{\mathrm{T}} Z_{j} A_{d i}
\end{array}\right]
\end{aligned}
$$


Then, for any $i, j \in \Re$, there exists a positive scalar $\delta$ such that

$$
\begin{aligned}
\sum_{i=1}^{r} h_{i}(\theta(k)) & \Phi_{s t l i i}+\sum_{i=1}^{r-1} \sum_{j=i+1}^{r} h_{i}(\theta(k)) \\
& \times h_{j}(\theta(k))\left(\Phi_{s t l i j}+\Phi_{s t l j i}\right)+\operatorname{diag}\{\delta I, 0\}<0 .
\end{aligned}
$$

Therefore, we have $\Delta V(k) \leq-\delta\|x(k)\|^{2}$ for all nonzero $x(k)$ 's, and the asymptotic stability is established.

Remark 2: In the derivation of Theorem 1, the slack variables $S_{1 i}$ and $S_{2 i}$ are introduced, the purpose of which is to reduce conservatism in the existing delay-dependent stability condition. From the proof of Theorem 1, we can see that no model transformation has been performed to the original system, and thus, no bounding technique has been employed to seek upper bounds of the inner product between vectors. Moreover, the inequalities in (6) and (7) are a set of LMIs, which can be readily solved using standard numerical software.

In Theorem 1, we have developed a delay-dependent stability condition for fuzzy systems with time-varying state delay. It is worth noting that most of the previous results for discrete time-delay systems assume the time delay to be constant. For the constant delay case, the lower and upper delay bounds in Assumption 1 become identical, i.e., $d_{m}=d_{M}=d$. Correspondingly, the time-varying delay system in (4) reduces to

$$
x(k+1)=\bar{A}(k) x(k)+\bar{A}_{d}(k) x(k-d) .
$$

With $d_{m}=d_{M}=d$ in (6) and (7), we obtain a delaydependent stability condition for the aforementioned constant fuzzy delay system in the following corollary.

Corollary 1: Consider the fuzzy time-delay system in (17). If there exist matrices $P_{i}>0, Q_{i}>0, Z_{i}>0$, and $S_{1 i}, S_{2 i}$, $i \in \Re$, such that (6) and (7) hold, where $\Pi_{\text {stlij }}$ is shown at the bottom of the page, with $\Pi_{1 i}=-P_{i}+Q_{i}+\operatorname{sym}\left(S_{1 i}\right)$, then the fuzzy time-delay system in (17) is asymptotically stable.

Note that the number of LMIs in Theorem 1 will increase with the number of fuzzy rules, which may increase the computational complexity. However, by a certain choice of matrices, we can get a tradeoff between conservativeness and computational complexity. If we take $P_{i}=P, Q_{i}=Q$, and $Z_{i}=Z$, in (6) and (7), the fuzzy Lyapunov function becomes a nonfuzzy one, and the following corollary can be obtained.

Corollary 2: The fuzzy time-delay system in (4) is asymptotically stable for time-varying delay $d(k)$ satisfying $0<d_{m} \leq$ $d(k) \leq d_{M}$, if there exist matrices $P>0, Q>0$, and $Z>0$, and matrices $S_{1}$ and $S_{2}$ satisfying the following LMI:

$$
\left[\begin{array}{ccccc}
\Pi_{1} & -S_{1}+S_{2}^{\mathrm{T}} & \sqrt{d_{M}} S_{1} & A_{i}^{\mathrm{T}} P & \sqrt{d_{M}}\left(A_{i}^{\mathrm{T}}-I\right) Z \\
* & -Q-\operatorname{sym}\left(S_{2}\right) & \sqrt{d_{M}} S_{2} & A_{d i}^{\mathrm{T}} P & \sqrt{d_{M}} A_{d i}^{\mathrm{T}} Z \\
* & * & -Z & 0 & 0 \\
* & * & * & -P & 0 \\
* & * & * & * & -Z
\end{array}\right]<0
$$

where $\Pi_{1}=-P+Q+\operatorname{sym}\left(S_{1}\right), \tau=d_{M}-d_{m}+1$.

\section{CONTROLler Synthesis}

In this section, Theorem 1 will be extended to design stabilizing state feedback and observer-based output feedback controllers for the fuzzy system in (3) with time-varying delay.

\section{A. State Feedback}

The purpose of this section is to design a controller, based on the PDC technique, such that the resultant closed-loop fuzzy time-varying delay system in (3) is asymptotically stable. Assume that the state is available for feedback control. The state feedback fuzzy controller is represented by the following rules.

\section{Controller Rule $i$ :}

IF $\theta_{1}(k)$ is $M_{i 1}$ and, .., and $\theta_{s}(k)$ is $M_{i s}$ THEN

$$
u(k)=K_{i} x(k), \quad i \in \Re
$$

where $K_{i} \in \mathbb{R}^{m \times n}, i \in \Re$, is the local control gain. Thus, the controller in (18) can be represented by the following input-output form:

$$
u(k)=\sum_{i=1}^{r} h_{i}(\theta(k)) K_{i} x(k)=\bar{K}(k) x(k) .
$$

By connecting (19) to system (3), we have the closed-loop fuzzy system

$$
x(k+1)=[\bar{A}(k)+\bar{B}(k) \bar{K}(k)] x(k)+\bar{A}_{d}(k) x(k-d(k)) .
$$

The design of the state feedback fuzzy control is to determine the feedback gains $K_{i}$ such that the closed-loop fuzzy system in (20) is asymptotically stable. The following theorem presents an LMI-based delay-dependent condition for the existence of a stabilizing state feedback controller.

Theorem 2: Consider the fuzzy time-delay system in (3) with time-varying delay $d(k)$ satisfying $0<d_{m} \leq d(k) \leq d_{M}$.

$$
\Pi_{s t l i j}=\left[\begin{array}{ccccc}
\Pi_{1 i} & -S_{1 i}+S_{2 i}^{\mathrm{T}} & \sqrt{d} S_{1 i} & A_{i}^{\mathrm{T}} P_{t} & \sqrt{d}\left(A_{i}^{\mathrm{T}}-I\right) Z_{j} \\
* & -Q_{s}-\operatorname{sym}\left(S_{2 i}\right) & \sqrt{d} S_{2 i} & A_{d i}^{\mathrm{T}} P_{t} & \sqrt{d} A_{d i}^{\mathrm{T}} Z_{j} \\
* & * & -Z_{l} & 0 & 0 \\
* & * & * & -P_{t} & 0 \\
* & * & * & * & -Z_{j}
\end{array}\right]
$$


A stabilizing controller in the form of (19) exists, such that the closed-loop fuzzy system in (20) is asymptotically stable, if there exist matrices $\tilde{P}_{i}>0, \tilde{Q}_{i}>0, \tilde{Z}_{i}>0$, and $\tilde{S}_{1 i}, \tilde{S}_{2 i}, G$, $\bar{K}_{i}$, satisfying the following LMIs for some scalar $\varepsilon>0$ :

$$
\begin{aligned}
\Gamma_{\text {stlii }}<0, & i, j, s, t, l \in \Re \\
\Gamma_{\text {stlij }}+\Gamma_{\text {stlji }}<0, & 1 \leq i<j \leq r
\end{aligned}
$$

where

$$
\begin{aligned}
& \Gamma_{s t l i j}=\left[\begin{array}{ccc}
\tilde{\Psi}_{i s}+\operatorname{sym}\left(\tilde{\Xi}_{2 i}\right) & \varepsilon \sqrt{d_{M}} \tilde{S}_{i} & \tilde{\Phi}_{1, i j} \\
* & -\tilde{Z}_{l} & 0 \\
* & * & \tilde{\Phi}_{2, j t}
\end{array}\right] \\
& \tilde{\Psi}_{i s}=\left[\begin{array}{cc}
-\tilde{P}_{i}+\tau \varepsilon^{-2} \tilde{Q}_{i} & 0 \\
0 & -\tilde{Q}_{s}
\end{array}\right] \quad \tau=d_{M}-d_{m}+1 \\
& \tilde{\Phi}_{1, i j}=\left[\begin{array}{cc}
G^{\mathrm{T}} A_{i}^{\mathrm{T}}+\bar{K}_{j}^{\mathrm{T}} B_{i}^{\mathrm{T}} \sqrt{d_{M}} G^{\mathrm{T}}\left(A_{i}^{\mathrm{T}}-I\right)+\sqrt{d_{M}} \bar{K}_{j}^{\mathrm{T}} B_{i}^{\mathrm{T}} \\
\varepsilon G^{\mathrm{T}} A_{d i}^{\mathrm{T}} & \varepsilon \sqrt{d_{M}} G^{\mathrm{T}} A_{d i}^{\mathrm{T}}
\end{array}\right] \\
& \tilde{\Xi}_{2 i}=\left[\begin{array}{ll}
\tilde{S}_{i} & -\varepsilon \tilde{S}_{i}
\end{array}\right] \quad \tilde{S}_{i}=\left[\begin{array}{c}
\tilde{S}_{1 i} \\
\tilde{S}_{2 i}
\end{array}\right] \\
& \tilde{\Phi}_{2, j t}=\left[\begin{array}{cc}
\tilde{P}_{t}-\operatorname{sym}(G) & 0 \\
0 & \tilde{Z}_{j}-\operatorname{sym}(\varepsilon G)
\end{array}\right] .
\end{aligned}
$$

Furthermore, if the aforementioned conditions are satisfied, the matrix gains $K_{j}$ of the controller are given by

$$
K_{j}=\bar{K}_{j} G^{-1} .
$$

Proof: Suppose that there exist matrices $\tilde{P}_{i}>0, \tilde{Q}_{i}>0$, and $\tilde{Z}_{i}>0$, and matrices $\tilde{S}_{1 i}, \tilde{S}_{2 i}$, and $G$ satisfying (21) and (22). Since $\tilde{P}_{t}>0, \tilde{Z}_{j}>0$, we have

$$
\begin{aligned}
{\left[\tilde{P}_{t}-G\right] \tilde{P}_{t}^{-1}\left[\tilde{P}_{t}-G\right]^{\mathrm{T}} } & \geq 0 \\
{\left[\tilde{Z}_{j}-\varepsilon G\right] \tilde{Z}_{j}^{-1}\left[\tilde{Z}_{j}-\varepsilon G\right]^{\mathrm{T}} } & \geq 0
\end{aligned}
$$

which imply that

$$
\begin{aligned}
-G \tilde{P}_{t}^{-1} G^{\mathrm{T}} & \leq \tilde{P}_{t}-G-G^{\mathrm{T}} \\
-\varepsilon^{2} G \tilde{Z}_{j}^{-1} G^{\mathrm{T}} & \leq \tilde{Z}_{j}-\varepsilon G-\varepsilon G^{\mathrm{T}} .
\end{aligned}
$$

Define

$$
F=\varepsilon G
$$

where $\varepsilon>0$ is a scalar; thus, (26) is equivalent to

$$
-F \tilde{Z}_{j}^{-1} F^{\mathrm{T}} \leq \tilde{Z}_{j}-F-F^{\mathrm{T}} .
$$

It is clear from (21) that $\tilde{P}_{t}-G-G^{\mathrm{T}}<0$. Since $\tilde{P}_{t}>0$, we have $G+G^{\mathrm{T}}>0$, which ensures that $G^{-1}$ exists. Define matrix $\Gamma_{11}=\operatorname{diag}\left\{G^{-1}, F^{-1}\right\}$ and $\Gamma_{1}=\operatorname{diag}\left\{\Gamma_{11}, F^{-1}, I\right\}$. By pre- and postmultiplying (21) and (22) by $\Gamma_{1}^{\mathrm{T}}$ and $\Gamma_{1}$, respectively, and by considering (25) and (28), we obtain

$$
\begin{aligned}
& \Gamma_{1}^{\mathrm{T}} \Gamma_{s t l i i} \Gamma_{1}<0 \\
& \Gamma_{1}^{\mathrm{T}} \Gamma_{s t l i j} \Gamma_{1}+\Gamma_{1}^{\mathrm{T}} \Gamma_{s t l j i} \Gamma_{1}<0, \quad 1 \leq i<j \leq r .
\end{aligned}
$$

Since

$$
\Gamma_{1}^{\mathrm{T}} \Gamma_{s t l i j} \Gamma_{1}=\left[\begin{array}{ccc}
\bar{\Psi}_{i s}+\operatorname{sym}\left(\bar{\Xi}_{2 i}\right) & \varepsilon \sqrt{d_{M} S_{i}} & \bar{\Phi}_{1, i j} \\
* & -F^{-T} \tilde{Z}_{l} F^{-1} & 0 \\
* & * & \bar{\Phi}_{2, j t}
\end{array}\right]
$$

where

$$
\begin{aligned}
& \bar{\Psi}_{i s}=\left[\begin{array}{cc}
-G^{-T} \tilde{P}_{i} G^{-1}+\tau F^{-T} \tilde{Q}_{i} F^{-1} & 0 \\
0 & -F^{-T} \tilde{Q}_{s} F^{-1}
\end{array}\right] \\
& \bar{\Xi}_{2 i}=\left[\begin{array}{ll}
\bar{S}_{i} & -\bar{S}_{i}
\end{array}\right] \quad \bar{S}_{i}=\left[\begin{array}{l}
G^{-T} \tilde{S}_{1 i} G^{-1} \\
G^{-T} \tilde{S}_{2 i} F^{-1}
\end{array}\right] \\
& \bar{\Phi}_{1, i j}=\left[\begin{array}{cc}
A_{i}^{\mathrm{T}}+K_{j}^{\mathrm{T}} B_{i}^{\mathrm{T}} & \sqrt{d_{M}}\left(A_{i}^{\mathrm{T}}-I\right)+\sqrt{d_{M}} K_{j}^{\mathrm{T}} B_{i}^{\mathrm{T}} \\
A_{d i}^{\mathrm{T}} & \sqrt{d_{M}} A_{d i}^{\mathrm{T}}
\end{array}\right] \\
& \bar{\Phi}_{2, j t}=\left[\begin{array}{cc}
-G \tilde{P}_{t}^{-1} G^{\mathrm{T}} & 0 \\
0 & -F \tilde{Z}_{j}^{-1} F^{\mathrm{T}}
\end{array}\right] \text {. }
\end{aligned}
$$

By defining

$$
\begin{aligned}
P_{i} & =G^{-T} \tilde{P}_{i} G^{-1} \quad Q_{i}=F^{-T} \tilde{Q}_{i} F^{-1} \quad Z_{j}=F^{-T} \tilde{Z}_{j} F^{-1} \\
S_{1 i} & =G^{-T} \tilde{S}_{1 i} G^{-1} \quad S_{2 i}=G^{-T} \tilde{S}_{2 i} F^{-1}
\end{aligned}
$$

we have

$$
\Gamma_{1}^{\mathrm{T}} \Gamma_{s t l i j} \Gamma_{1}=\left[\begin{array}{ccc}
\operatorname{sym}\left(\Xi_{2 i}\right)+\Psi_{i s} & \sqrt{d_{M}} S_{i} & \bar{\Phi}_{1, i j t} \\
* & -Z_{l} & 0 \\
* & * & \hat{\Phi}_{2, j t}
\end{array}\right]
$$

where

$$
\hat{\Phi}_{2, j t}=\left[\begin{array}{cc}
-P_{t}^{-1} & 0 \\
0 & -Z_{j}^{-1}
\end{array}\right]
$$

and $\Xi_{2 i}, \Psi_{i s}$, and $S_{i}$ are defined in (9). Define matrix $\Theta_{t j}=$ $\operatorname{diag}\left\{I, P_{t}, Z_{j}\right\}$; by pre- and postmultiplying (32) by $\Theta_{t j}^{\mathrm{T}}$ and $\Theta_{t j}$, we obtain

$$
\Theta_{t j}^{\mathrm{T}} \Gamma_{1}^{\mathrm{T}} \Gamma_{s t l i j} \Gamma_{1} \Theta_{t j}=\left[\begin{array}{ccc}
\operatorname{sym}\left(\Xi_{2 i}\right)+\Psi_{i s} & \sqrt{d_{M}} S_{i} & \bar{\Phi}_{1, i j t} \\
* & -Z_{l} & 0 \\
* & * & \Phi_{2, j t}
\end{array}\right]
$$

where $\Phi_{2, j t}$ is defined in (9). Therefore, by Schur complement, we can obtain that (29) and (30) yield (6) and (7) with $A_{i}$ replaced by $A_{i}+B_{i} K_{j}$, which means that there exist matrices $P_{i}>0, Q_{i}>0, Z_{i}>0$, and $S_{1 i}, S_{2 i}, i \in \Re$, satisfying (6) and (7), and thus, the controller gains defined in (24) render the closed-loop fuzzy delay system in (20) asymptotically stable.

Remark 3: With the introduction of the new additional matrices $G$ and $F$, we obtain a sufficient condition in which the Lyapunov matrices are not involved in any product with the system matrices. It is noted that the introduced matrices $G$ and $F$ are not even constrained to be symmetric. This feature enables us to derive a less conservative condition due to the extra degrees of freedom. This technique has been used in [4] to handle the corresponding problem. We let $F=\varepsilon G$ with $\varepsilon$ being a positive scalar, which may be adjusted to reduce the conservatism in the controller design. More specifically, 
in numerical implementation of Theorem 2, due to the nonmonotonic behavior of $\varepsilon$ in the feasibility of the conditions in Theorem 2, its value has to be tuned over an interval so that a less conservative design can be obtained.

\section{B. Observer-Based Control}

A design approach of the state feedback controller is developed in the previous section. Its main drawback comes from the requirement of accessing all the state variables. In some practical applications, not all the state variables are available. Therefore, it is necessary to design an output feedback controller. In this section, we consider an observer-based output feedback controller for the system in (3). As in the case of state feedback controller design, the PDC concept is employed to arrive at the following output feedback fuzzy observer.

\section{Observer Rule $i$ :}

IF $\theta_{1}(k)$ is $M_{i 1}$ and, ..., and $\theta_{s}(k)$ is $M_{i s}$

\section{THEN}

$$
\begin{aligned}
\hat{x}(k+1)= & A_{i} \hat{x}(k)+A_{d i} \hat{x}(k-d(k)) \\
& +B_{i} u(k)+L_{i}(y(k)-\hat{y}(k)) \\
\hat{y}(k)= & C_{i} \hat{x}(k)+C_{d i} \hat{x}(k-d(k)), \quad i \in \Re
\end{aligned}
$$

where $L_{i} \in \mathbb{R}^{n \times q}$ denotes the observer gains to be determined. $\hat{x}(k)=\phi(k), k=-d_{M},-d_{M}+1, \ldots, 0$, is the initial condition sequence of the observer. As in the case of the state feedback controller design, the following PDC fuzzy controller is proposed to stabilize system (3), i.e.,

$$
u(k)=\sum_{i=1}^{r} h_{i}(\theta(k)) K_{i} \hat{x}(k)=\bar{K}(k) \hat{x}(k)
$$

where $K_{i} \in \mathbb{R}^{m \times n}$ denotes the controller gains to be determined. Let us denote the estimation error as $e(k)=x(k)-$ $\hat{x}(k)$. From (3), (33), and (34), the augmented closed-loop fuzzy system can be written as the following form:

$$
\begin{aligned}
\xi(k+1)= & \sum_{i=1}^{r} h_{i}(\theta(k)) \sum_{j=1}^{r} h_{j}(\theta(k)) \\
& \times\left[A_{a i j} \xi(k)+A_{d a i j} \xi(k-d(k))\right] \\
\xi(k)= & \varphi_{a}(k), \quad k=-d_{M},-d_{M}+1, \ldots, 0
\end{aligned}
$$

where

$$
\begin{aligned}
\xi(k) & =\left[\begin{array}{l}
x(k) \\
e(k)
\end{array}\right] \quad \varphi_{a}(k)=\left[\begin{array}{c}
\varphi(k) \\
\phi(k)-\varphi(k)
\end{array}\right] \\
A_{a i j} & =\left[\begin{array}{cc}
A_{i}+B_{i} K_{j} & -B_{i} K_{j} \\
0 & A_{i}-L_{i} C_{j}
\end{array}\right] \\
A_{d a i j} & =\left[\begin{array}{cc}
A_{d i} & 0 \\
0 & A_{d i}-L_{i} C_{d j}
\end{array}\right]
\end{aligned}
$$

with its compact form given by

$$
\xi(k+1)=\bar{A}_{a}(k) \xi(k)+\bar{A}_{d a}(k) \xi(k-d(k))
$$

where

$$
\begin{aligned}
\bar{A}_{a}(k) & =\left[\begin{array}{cc}
\bar{A}(k)+\bar{B}(k) \bar{K}(k) & -\bar{B}(k) \bar{K}(k) \\
0 & \bar{A}(k)-\bar{L}(k) \bar{C}(k)
\end{array}\right] \\
\bar{A}_{d a}(k) & =\left[\begin{array}{cc}
\bar{A}_{d}(k) & \bar{A}_{d}(k)-\bar{L}(k) \bar{C}_{d}(k)
\end{array}\right] \\
0 & \\
\bar{L}(k) & =\sum_{i=1}^{r} h_{i}(\theta(k)) L_{i} .
\end{aligned}
$$

By applying Theorem 2 to system (35), we can obtain the following result, which presents an LMI-based delay-dependent condition for the existence of a stabilizing fuzzy observer-based output feedback controller.

Theorem 3: Consider the fuzzy system in (3) with the timevarying delay $d(k)$ satisfying $0<d_{m} \leq d(k) \leq d_{M}$. If, for some scalar $\varepsilon>0$, there exist $n \times n$ matrices $\tilde{P}_{1 i}>0, \check{P}_{2 i}$, $\check{P}_{3 i}>0, \tilde{Z}_{1 i}>0, \check{Z}_{2 i}, \check{Z}_{3 i}>0, \tilde{Q}_{1 i}>0, \check{Q}_{2 i}, \check{Q}_{3 i}>0$ and $\tilde{S}_{11 i}, \check{S}_{12 i}, \check{S}_{13 i}, \check{S}_{14 i}, \tilde{S}_{21 i}, \check{S}_{22 i}, \check{S}_{23 i}, \check{S}_{24 i}, G_{1}, G_{2}, K_{i}, L_{i}$, $i \in \Re$, satisfying the LMIs of (21) and (22), where $\Gamma_{\text {stlij }}$ is replaced by (36) and (37) shown at the bottom of the next page, then there exists a fuzzy controller of the form in (33) and (34) such that the closed-loop fuzzy system in (35) is asymptotically stable. Furthermore, the observer gain matrices are given by

$$
K_{j}=\bar{K}_{j} G_{1}^{-1} \quad L_{i}=G_{2}^{-T} \bar{L}_{i}, \quad i, j \in \Re .
$$

Proof: By Theorem 2, the system in (35) is asymptotically stable, if there exist $2 n \times 2 n$ matrices $\tilde{P}_{i}>0, \tilde{Q}_{i}>0, \tilde{Z}_{i}>0$, and $G, \tilde{S}_{1 i}, \tilde{S}_{2 i}$ satisfying (21) and (22) with $A_{i}+B_{i} K_{j}$ and $A_{d i}$ replaced by $A_{a i j}$ and $A_{d a i j}$, respectively. Let $\tilde{P}_{i}, \tilde{Z}_{i}, \tilde{Q}_{i}$, $\tilde{S}_{1 i}$, and $\tilde{S}_{2 i}$ be respectively partitioned as

$$
\begin{array}{rlr}
\tilde{P}_{i}=\left[\begin{array}{cc}
\tilde{P}_{1 i} & \tilde{P}_{2 i} \\
* & \tilde{P}_{3 i}
\end{array}\right] & \tilde{S}_{1 i}=\left[\begin{array}{cc}
\tilde{S}_{11 i} & \tilde{S}_{12 i} \\
\tilde{S}_{13 i} & \tilde{S}_{14 i}
\end{array}\right] \\
\tilde{Q}_{i}=\left[\begin{array}{cc}
\tilde{Q}_{1 i} & \tilde{Q}_{2 i} \\
* & \tilde{Q}_{3 i}
\end{array}\right] & \tilde{S}_{2 i}=\left[\begin{array}{cc}
\tilde{S}_{21 i} & \tilde{S}_{22 i} \\
\tilde{S}_{23 i} & \tilde{S}_{24 i}
\end{array}\right] \\
\tilde{Z}_{i}=\left[\begin{array}{cc}
\tilde{Z}_{1 i} & \tilde{Z}_{2 i} \\
* & \tilde{Z}_{3 i}
\end{array}\right] &
\end{array}
$$

where $\tilde{P}_{1 i}, \tilde{P}_{3 i}, \tilde{Q}_{1 i}, \tilde{Q}_{3 i}, \tilde{Z}_{1 i}$, and $\tilde{Z}_{3 i}$ are $n \times n$ real symmetric and positive definite, and $\tilde{P}_{2 i}, \tilde{Q}_{2 i}, \tilde{Z}_{2 i}, \tilde{S}_{11 i}, \tilde{S}_{12 i}, \tilde{S}_{13 i}$, $\tilde{S}_{14 i}, \tilde{S}_{21 i}, \tilde{S}_{22 i}, \tilde{S}_{23 i}, \tilde{S}_{24 i} \in \mathbb{R}^{n \times n}$.

By considering the inequalities in (21) and (22), it is clear from $\check{\Phi}_{2, j t}$ defined in (37) that

$$
\tilde{P}_{1 t}-G_{1}-G_{1}^{\mathrm{T}}<0 \quad \check{P}_{3 t}-G_{2}-G_{2}^{\mathrm{T}}<0 .
$$

Since $\tilde{P}_{1 t}>0$ and $\check{P}_{3 t}>0$, we have $G_{1}+G_{1}^{\mathrm{T}}>0$ and $G_{2}+$ $G_{2}^{\mathrm{T}}>0$, which imply that $G_{1}^{-1}$ and $G_{2}^{-1}$ exist. In order 
to obtain an LMI-based stabilization condition, let $G$ be by letting constructed as

$$
G=\left[\begin{array}{cc}
G_{1} & G_{2}^{-1} \\
0 & G_{2}^{-1}
\end{array}\right]
$$

$$
\begin{array}{ll}
\check{P}_{2 i}=\tilde{P}_{2 i} G_{2} & \check{P}_{3 i}=G_{2}^{\mathrm{T}} \tilde{P}_{3 i} G_{2} \quad \check{Q}_{2 i}=\tilde{Q}_{2 i} G_{2} \\
\check{Z}_{2 j}=\tilde{Z}_{2 j} G_{2} & \check{Z}_{3 j}=G_{2}^{\mathrm{T}} \tilde{Z}_{3 j} G_{2} \quad \check{Q}_{3 i}=G_{2}^{\mathrm{T}} \tilde{Q}_{3 i} G_{2} \\
\check{S}_{l 2 i}=\tilde{S}_{l 2 i} G_{2} & \check{S}_{l 3 i}=G_{2}^{\mathrm{T}} \tilde{S}_{l 3 i} \quad \check{S}_{l 4 i}=G_{2}^{\mathrm{T}} \tilde{S}_{l 4 i} G_{2}, \quad l=1,2
\end{array}
$$

Following the earlier notation, we let

$$
\begin{aligned}
& {\left[\begin{array}{ccc}
\tilde{P}_{n}(k) & \tilde{Q}_{n}(k) & \tilde{Z}_{n}(k) \\
\tilde{S}_{1 m}(k) & \tilde{S}_{2 m}(k) & 0
\end{array}\right]} \\
& =\sum_{i=1}^{r} h_{i}(\theta(k))\left[\begin{array}{ccc}
\tilde{P}_{n i} & \tilde{Q}_{n i} & \tilde{Z}_{n i} \\
\tilde{S}_{1 m i} & \tilde{S}_{2 m i} & 0
\end{array}\right] \\
& n=1,2,3, \quad m=1,2,3,4 .
\end{aligned}
$$

and by defining

$$
\bar{K}_{j}=K_{j} G_{1} \quad \bar{L}_{i}=G_{2}^{\mathrm{T}} L_{i}, \quad i, j \in \Re
$$

we obtain (36). The proof is completed.

\section{ILLUSTRATIVE EXAMPLES}

Define $A_{G K}=A_{i} G_{1}+B_{i} K_{j} G_{1}, \quad A_{L}=A_{i}-L_{i} C_{j}$, and $A_{d L}=A_{d i}-L_{i} C_{d j}$. Then, from $\Gamma_{s t l i j}$ in (23) with $A_{i}+B_{i} K_{j}$ and $A_{d i}$ replaced by $A_{a i j}$ and $A_{d a i j}$, respectively, we have

$$
\left[\begin{array}{ccc}
\hat{\Psi}_{i s}+\operatorname{sym}\left(\hat{\Xi}_{2 i}\right) & \varepsilon \sqrt{d_{M}} \hat{S}_{i} & \hat{\Phi}_{1, i j} \\
* & -\tilde{Z}_{l} & 0 \\
* & * & \hat{\Phi}_{2, i t}
\end{array}\right]<0
$$

where the expressions $\hat{\Psi}_{i s}, \hat{\Xi}_{2 i}, \hat{\Phi}_{1, i j}$, and $\hat{\Phi}_{2, j t}$ are shown at the bottom of the next page.

Define matrix $\Gamma_{2}=\operatorname{diag}\left\{I, G_{2}, I, G_{2}, I, G_{2}, I, G_{2}, I, G_{2}\right\}$. By pre- and postmultiplying (38) by $\Gamma_{2}^{\mathrm{T}}$ and $\Gamma_{2}$, respectively,

In this section, two examples are provided to illustrate the effectiveness and the advantages of the methods developed previously. We first use a numerical example to show the advantages of the proposed stability condition in this paper. The second example is utilized to illustrate the effectiveness of the proposed stabilization methods.

Example 1: Consider a discrete-time fuzzy delay system in (3) with the following matrices:

$$
\begin{array}{rlrl}
A_{1} & =\left[\begin{array}{cc}
-0.291 & 1 \\
0 & 0.95
\end{array}\right] & A_{2}=\left[\begin{array}{cc}
-0.1 & 0 \\
1 & -0.2
\end{array}\right] \\
A_{d 1}=\left[\begin{array}{cc}
0.012 & 0.014 \\
0 & 0.015
\end{array}\right] & A_{d 2}=\left[\begin{array}{cc}
0.01 & 0 \\
0.01 & 0.015
\end{array}\right] .
\end{array}
$$

$$
\begin{aligned}
& \Gamma_{\text {stlij }}=\left[\begin{array}{ccc}
\check{\Psi}_{i s}+\operatorname{sym}\left(\check{\Xi}_{2 i}\right) & \varepsilon \sqrt{d_{M}} \check{S}_{i} & \check{\Phi}_{1, i j} \\
* & -\check{Z}_{l} & 0 \\
* & * & \check{\Phi}_{2, i t}
\end{array}\right] \\
& \check{\Psi}_{i s}=\left[\begin{array}{cccc}
-\tilde{P}_{1 i}+\tau \varepsilon^{-2} \tilde{Q}_{1 i} & -\check{P}_{2 i}+\tau \varepsilon^{-2} \check{Q}_{2 i} & 0 & 0 \\
* & -\check{P}_{3 i}+\tau \varepsilon^{-2} \check{Q}_{3 i} & 0 & 0 \\
* & * & -\tilde{Q}_{1 s} & -\check{Q}_{2 s} \\
* & * & * & -\check{Q}_{3 s}
\end{array}\right] \\
& \tau=d_{M}-d_{m}+1 \quad \check{\Xi}_{2 i}=\left[\begin{array}{ll}
\check{S}_{i} & -\varepsilon \check{S}_{i}
\end{array}\right] \\
& \check{S}_{i}=\left[\begin{array}{cc}
\tilde{S}_{11 i} & \check{S}_{12 i} \\
\check{S}_{13 i} & \check{S}_{14 i} \\
\widetilde{S}_{21 i} & \check{S}_{22 i} \\
\check{S}_{23 i} & \check{S}_{24 i}
\end{array}\right] \quad \check{Z}_{l}=\left[\begin{array}{cc}
\tilde{Z}_{1 l} & \check{Z}_{2 l} \\
* & \check{Z}_{3 l}
\end{array}\right] \\
& \check{\Phi}_{1, i j}=\left[\begin{array}{cccc}
G_{1}^{\mathrm{T}} A_{i}^{\mathrm{T}}+\bar{K}_{j}^{\mathrm{T}} B_{i}^{\mathrm{T}} & 0 & \sqrt{d_{M}}\left(G_{1}^{\mathrm{T}} A_{i}^{\mathrm{T}}+\bar{K}_{j}^{\mathrm{T}} B_{i}^{\mathrm{T}}-G_{1}^{\mathrm{T}}\right) & 0 \\
A_{i}^{\mathrm{T}} & A_{i}^{\mathrm{T}} G_{2}-C_{j}^{\mathrm{T}} \bar{L}_{i}^{\mathrm{T}} & \sqrt{d_{M}}\left(A_{i}^{\mathrm{T}}-I\right) & \sqrt{d_{M}}\left(A_{i}^{\mathrm{T}} G_{2}-C_{j}^{\mathrm{T}} \bar{L}_{i}^{\mathrm{T}}-G_{2}\right) \\
\varepsilon G_{1}^{\mathrm{T}} A_{d i}^{\mathrm{T}} & 0 & \varepsilon \sqrt{d_{M}} G_{1}^{\mathrm{T}} A_{d i}^{\mathrm{T}} & 0 \\
\varepsilon A_{d i}^{\mathrm{T}} & \varepsilon A_{d i}^{\mathrm{T}} G_{2}-\varepsilon C_{d j}^{\mathrm{T}} \bar{L}_{i}^{\mathrm{T}} & \varepsilon \sqrt{d_{M}} A_{d i}^{\mathrm{T}} & \varepsilon \sqrt{d_{M}} A_{d i}^{\mathrm{T}} G_{2}-C_{d j}^{\mathrm{T}} \bar{L}_{i}^{\mathrm{T}}
\end{array}\right] \\
& \check{\Phi}_{2, j t}=\left[\begin{array}{cccc}
\tilde{P}_{1 t}-\operatorname{sym}\left(G_{1}\right) & \check{P}_{2 t}-I & 0 & 0 \\
* & \check{P}_{3 t}-\operatorname{sym}\left(G_{2}\right) & 0 & 0 \\
* & * & \tilde{Z}_{1 j}-\operatorname{sym}\left(\varepsilon G_{1}\right) & \check{Z}_{2 j}-\varepsilon I \\
* & * & * & \check{Z}_{3 j}-\operatorname{sym}\left(\varepsilon G_{2}\right)
\end{array}\right]
\end{aligned}
$$


TABLE I

$d_{M}$ FOR DIFFERENT VALUES OF $d_{m}$

\begin{tabular}{l|c|c|c|c|c|c}
\hline lower delay bound $d_{m}$ & 1 & 3 & 5 & 8 & 10 & 12 \\
\hline upper delay bound $d_{M}$ & 13 & 14 & 16 & 18 & 20 & 21 \\
\hline
\end{tabular}

In this example, $d(k)$ presents a time-varying state delay, and Table I shows the upper delay bounds in terms of the feasibility of (6) and (7) for the different values of lower delay bounds.

More specifically, let $d_{m}=3$; by implementing Theorem 1 numerically, it is found that the upper delay bound $d_{M}=14$ such that the aforementioned system is asymptotically stable for all $0<d_{m} \leq d(K) \leq d_{M}$

$$
\begin{aligned}
P_{1} & =\left[\begin{array}{cc}
0.0059 & -0.0046 \\
-0.0046 & 0.0145
\end{array}\right] \\
P_{2} & =\left[\begin{array}{cc}
0.0252 & -0.0126 \\
-0.0126 & 0.0123
\end{array}\right] \\
Q_{1} & =10^{-4} \cdot\left[\begin{array}{cc}
0.5115 & -0.1732 \\
-0.1732 & 0.3954
\end{array}\right] \\
Q_{2} & =10^{-4} \cdot\left[\begin{array}{cc}
0.7925 & -0.4955 \\
-0.4955 & 0.7665
\end{array}\right] \\
Z_{1} & =10^{-3} \cdot\left[\begin{array}{cc}
0.0099 & 0.0184 \\
0.0184 & 0.9745
\end{array}\right] \\
Z_{2} & =10^{-4} \cdot\left[\begin{array}{ll}
0.0112 & 0.0403 \\
0.0403 & 0.4738
\end{array}\right] .
\end{aligned}
$$

Example 2: Consider the following Hénon system:

$$
\begin{aligned}
x_{1}(k+1)= & -\left\{c x_{1}(k)+(1-c) x_{1}(k-d(k))\right\}^{2} \\
& +0.3 x_{2}(k)+1.4+u(k) \\
x_{2}(k+1)= & c x_{1}(k)+(1-c) x_{1}(k-d(k)) \\
y(k)= & c x_{1}(k)+(1-c) x_{1}(k-d(k))
\end{aligned}
$$

where the constant $c \in[0,1]$ is the retarded coefficient.
Let $\theta(k)=c x_{1}(k)+(1-c) x_{1}(k-d(k))$. Assume that $\theta(k) \in[-m, m], m>0$. By using the same procedure as in [29], the nonlinear term $\theta^{2}(k)$ can be exactly represented as

$$
\theta^{2}(k)=h_{1}(\theta(k))(-m) \theta(k)+h_{2}(\theta(k)) m \theta(k)
$$

where $h_{1}(\theta(k)), h_{2}\left(\theta(k) \in[0,1]\right.$, and $h_{1}(\theta(k))+h_{2}(\theta(k))=1$. By solving the equations, the membership functions $h_{1}(\theta(k))$ and $h_{2}(\theta(k))$ are obtained as

$h_{1}(\theta(k))=\frac{1}{2}\left(1-\frac{\theta(k)}{m}\right) \quad h_{2}(\theta(k))=\frac{1}{2}\left(1+\frac{\theta(k)}{m}\right)$.

It can be seen from the aforementioned expressions that $h_{1}(\theta(k))=1$ and $h_{2}(\theta(k))=0$ when $\theta(k)$ is $-m$ and that $h_{1}(\theta(k))=0$ and $h_{2}(\theta(k))=1$ when $\theta(k)$ is $m$. Then, the nonlinear system in (39) can be approximately represented by the following T-S fuzzy model:

\section{Plant Rule 1:}

IF $\theta(k)$ is $-m$, THEN

$$
\begin{aligned}
x(k+1) & =A_{1} x(k)+A_{d 1} x(k-d(k))+B_{1} u^{*}(k) \\
y(k) & =C_{1} x(k)+C_{d 1} x(k-d(k)) .
\end{aligned}
$$

\section{Plant Rule 2:}

IF $\theta(k)$ is $m$, THEN

$$
\begin{aligned}
x(k+1) & =A_{2} x(k)+A_{d 2} x(k-d(k))+B_{2} u^{*}(k) \\
y(k) & =C_{2} x(k)+C_{d 2} x(k-d(k))
\end{aligned}
$$

$$
\begin{aligned}
& \hat{\Psi}_{i s}=\left[\begin{array}{cccc}
-\tilde{P}_{1 i}+\tau \varepsilon^{-2} \tilde{Q}_{1 i} & -\tilde{P}_{2 i}+\tau \varepsilon^{-2} \tilde{Q}_{2 i} & 0 & 0 \\
* & -\tilde{P}_{3 i}+\tau \varepsilon^{-2} \tilde{Q}_{3 i} & 0 & 0 \\
0 & 0 & -\tilde{Q}_{1 s} & -\tilde{Q}_{2 s} \\
0 & 0 & * & -\tilde{Q}_{3 s}
\end{array}\right] \\
& \hat{\Xi}_{2 i}=\left[\begin{array}{ll}
\tilde{S}_{i} & -\varepsilon \tilde{S}_{i}
\end{array}\right] \quad \tilde{S}_{i}=\left[\begin{array}{cc}
\tilde{S}_{11 i} & \tilde{S}_{12 i} \\
\tilde{S}_{13 i} & \tilde{S}_{14 i} \\
\tilde{S}_{21 i} & \tilde{S}_{22 i} \\
\tilde{S}_{23 i} & \tilde{S}_{24 i}
\end{array}\right] \quad \tilde{Z}_{l}=\left[\begin{array}{cc}
\tilde{Z}_{1 l} & \tilde{Z}_{2 l} \\
* & \tilde{Z}_{3 l}
\end{array}\right] \\
& \hat{\Phi}_{1, i j}=\left[\begin{array}{cccc}
A_{G K}^{\mathrm{T}} & 0 & \sqrt{d_{M}}\left(A_{G K}^{\mathrm{T}}-G_{1}^{\mathrm{T}}\right) & 0 \\
G_{2}^{-T} A_{i}^{\mathrm{T}} & G_{2}^{-T} A_{L}^{\mathrm{T}} & \sqrt{d_{M}} G_{2}^{-T}\left(A_{i}^{\mathrm{T}}-I\right) & \sqrt{d_{M}} G_{2}^{-T}\left(A_{L}^{\mathrm{T}}-I\right) \\
\varepsilon G_{1}^{\mathrm{T}} A_{d i}^{\mathrm{T}} & 0 & \varepsilon \sqrt{d_{M}} G_{1}^{\mathrm{T}} A_{d i}^{\mathrm{T}} & 0 \\
\varepsilon G_{2}^{-T} A_{d i}^{\mathrm{T}} & \varepsilon G_{2}^{-T} A_{d L}^{\mathrm{T}} & \varepsilon \sqrt{d_{M}} G_{2}^{-T} A_{d i}^{\mathrm{T}} & \varepsilon \sqrt{d_{M}} G_{2}^{-T} A_{d L}^{\mathrm{T}}
\end{array}\right] \\
& \hat{\Phi}_{2, j t}=\left[\begin{array}{cccc}
\tilde{P}_{1 t}-\operatorname{sym}\left(G_{1}\right) & \tilde{P}_{2 t}-G_{2}^{-1} & 0 & 0 \\
* & \tilde{P}_{3 t}-\operatorname{sym}\left(G_{2}^{-1}\right) & 0 & 0 \\
* & * & \tilde{Z}_{1 j}-\operatorname{sym}\left(\varepsilon G_{1}\right) & \tilde{Z}_{2 j}-\varepsilon G_{2}^{-1} \\
* & * & * & \tilde{Z}_{3 j}-\operatorname{sym}\left(\varepsilon G_{2}^{-1}\right)
\end{array}\right]
\end{aligned}
$$




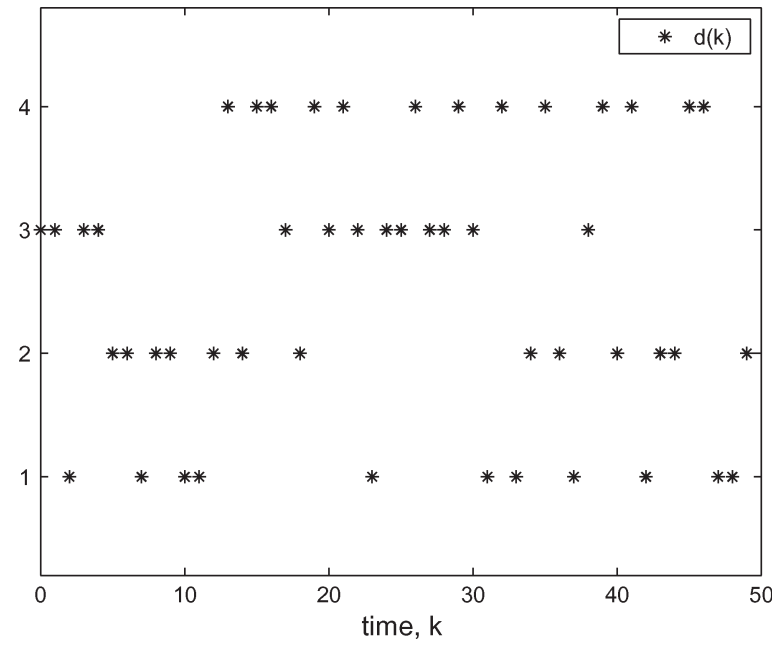

Fig. 1. Time-varying delays: State-feedback case.

where $u^{*}(k)=1.4+u(k)$ and

$$
\begin{aligned}
& A_{1}=\left[\begin{array}{cc}
c m & 0.3 \\
c & 0
\end{array}\right] \quad A_{2}=\left[\begin{array}{cc}
-c m & 0.3 \\
c & 0
\end{array}\right] \\
& A_{d 1}=\left[\begin{array}{cc}
(1-c) m & 0 \\
1-c & 0
\end{array}\right] \quad A_{d 2}=\left[\begin{array}{cc}
-(1-c) m & 0 \\
1-c & 0
\end{array}\right] \\
& B_{1}=B_{2}=\left[\begin{array}{l}
1 \\
0
\end{array}\right] \\
& C_{1}=C_{2}=\left[\begin{array}{ll}
c & 0
\end{array}\right] \quad C_{d 1}=C_{d 2}=\left[\begin{array}{ll}
1-c & 0
\end{array}\right] .
\end{aligned}
$$

In the example, $x^{\mathrm{T}}(k)=\left[x_{1}^{\mathrm{T}}(k) x_{2}^{\mathrm{T}}(k)\right]^{\mathrm{T}}, c=0.8, m=2$, and $d(k)$ represents a time-varying state delay. For simulation purposes, the initial condition is assumed to be $\varphi(k)=$ $\left[\mathrm{e}^{k / d_{M}} 0\right]$ for all $k=-d_{M},-d_{M}+1, \ldots, 0$. Here, our purpose is to design state feedback controller and observer-based output feedback controller in the form of (18), (33), and (34) such that the resulting closed-loop system is asymptotically stable.

\section{A. State Feedback Case}

Assume that the state is available. With the choice of $\varepsilon=10$, it is found that the aforementioned system is asymptotically stable for all $d_{M} \leq 4$. When $d_{M}=4$, Theorem 2 yields the fuzzy controller gains

$$
K_{1}=\left[\begin{array}{ll}
-1.4649 & -0.2902
\end{array}\right] \quad K_{2}=\left[\begin{array}{ll}
1.7958 & -0.3010
\end{array}\right] .
$$

In addition, let the delay $d(k)$ change randomly between $d_{m}=$ 1 and $d_{M}=4$ (see Fig. 1). To illustrate the behavior of the control action, Fig. 2 shows the behavior of the open-loop state response with time delays $d_{m}=1$ and $d_{M}=4$, from which we observe that the open-loop system is not guaranteed to be asymptotically stable. In addition, the state response of the closed-loop system is shown in Fig. 3, where the control input
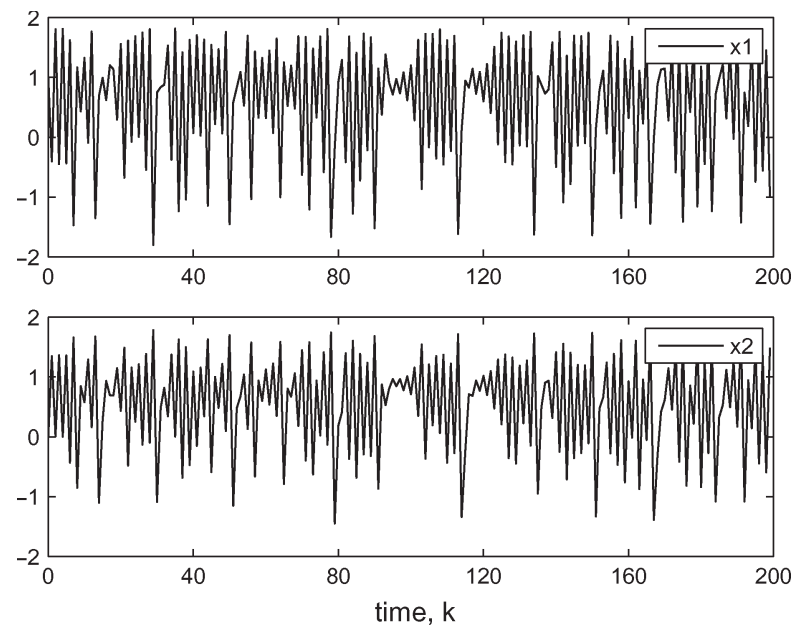

Fig. 2. State response of open-loop system.
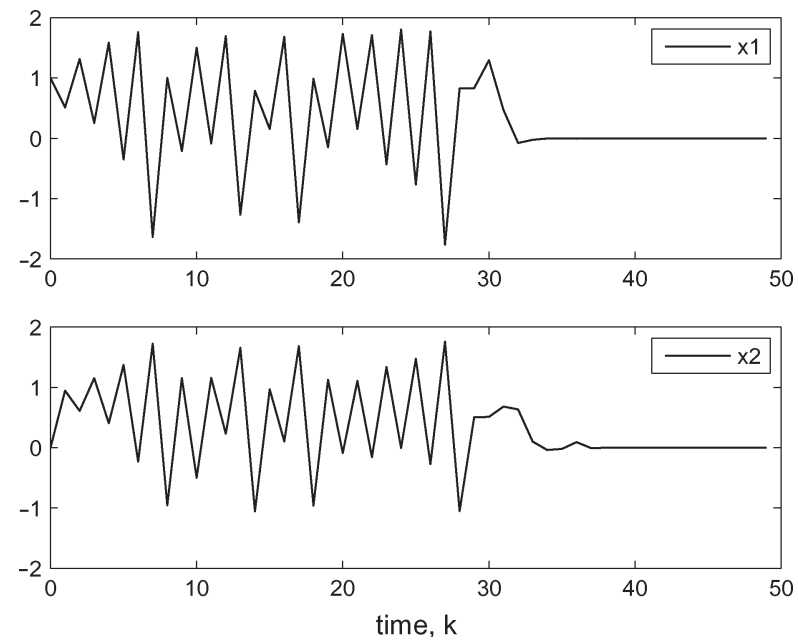

Fig. 3. State response: State-feedback case.

is added at $k \geq 30$. It is shown in Fig. 3 that the closed-loop system is asymptotically stable under the aforementioned state feedback controller.

\section{B. Observer-Based Output Feedback Case}

Assume that only $x_{1}(k)$ is measurable. By choosing $\varepsilon=50$, it can be found that the LMIs of Theorem 3 have a solution for all $d_{M} \leq 5$. When $d_{M}=5$, Theorem 3 yields the observer and controller gains

$$
\begin{aligned}
L_{1} & =\left[\begin{array}{l}
1.9930 \\
0.9999
\end{array}\right] & L_{2} & =\left[\begin{array}{c}
-2.0071 \\
0.9999
\end{array}\right] \\
K_{1} & =\left[\begin{array}{lll}
-1.5622 & -0.2966
\end{array}\right] & K_{2} & =\left[\begin{array}{ll}
1.6822 & -0.3015
\end{array}\right] .
\end{aligned}
$$

The simulation results are based on the same initial condition as aforementioned. In addition, let the delay $d(k)$ change randomly between $d_{m}=1$ and $d_{M}=5$, which is shown in Fig. 4. By applying the fuzzy controller (33) and (34) with 


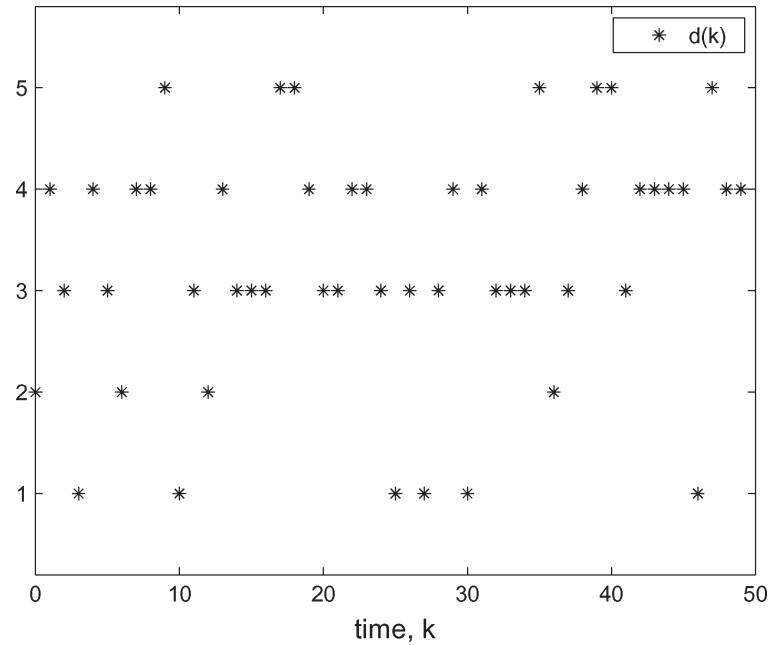

Fig. 4. Time-varying delays: Observer-based output-feedback case.

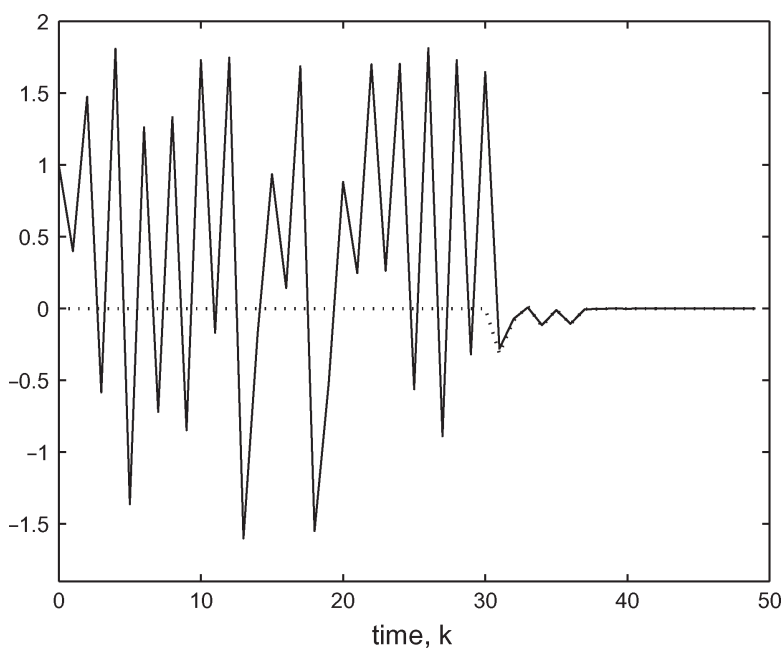

Fig. 5. State responses of $x_{1}$ and $\hat{x}_{1}$.

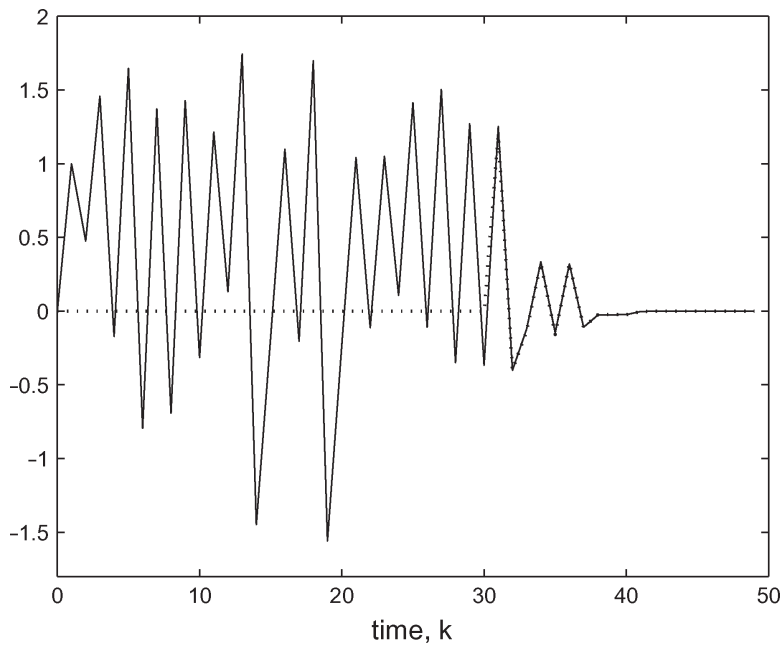

Fig. 6. State responses of $x_{2}$ and $\hat{x}_{2}$. the aforementioned matrices to the system in (39), the control results are shown in Figs. 5 and 6, where the control input is added at $k \geq 30$. In the two figures, the solid lines present the state response, and the dotted lines denote the corresponding state of fuzzy observer. The simulation results indicate that the designed observer-based output feedback controller can stabilize the Hénon system with time-varying state delay.

\section{CONCLUSION}

The stability analysis and stabilization for discrete-time T-S fuzzy systems with time-varying state delay have been investigated in this paper. First, by defining a new fuzzy Lyapunov functions and by making use of novel techniques, an improved delay-dependent stability condition has been established in terms of LMIs, which is dependent on the lower and upper delay bounds. The merit of the proposed condition lies in its reduced conservatism, which is achieved by circumventing the utilization of some bounding inequalities for cross products between two vectors. Then, a delay-dependent stabilization approach based on a PDC scheme has been provided for closedloop fuzzy systems. Both the state feedback and observerbased output feedback control cases have been considered. Finally, two illustrative examples are provided to demonstrate the effectiveness of the approaches proposed in this paper. The main results in this paper may be further extended to fuzzy systems with Lipschitz-like nonlinearities [18], [19].

\section{REFERENCES}

[1] Y. Y. Cao and P. M. Frank, "Analysis and synthesis of nonlinear time-delay systems via fuzzy control approach," IEEE Trans. Fuzzy Syst., vol. 8, no. 2, pp. 200-211, Apr. 2000.

[2] C. Chen, G. Feng, and X. Guan, "Delay-dependent stability analysis and controller synthesis for discrete-time T-S fuzzy systems with time delays," IEEE Trans. Fuzzy Syst., vol. 13, no. 5, pp. 630-643, Oct. 2005.

[3] J. Chen, D. M. Xu, and B. Shafai, "On sufficient conditions for stability independent of delay," IEEE Trans. Autom. Control, vol. 40, no. 9, pp. 1675-1680, Sep. 1995.

[4] M. C. de Oliveira, J. Bernussou, and J. C. Geromel, "A new discrete-time robust stability condition," Syst. Control Lett., vol. 37, no. 4, pp. 261-265, Jul. 1999.

[5] G. Feng, "Stability analysis of discrete-time fuzzy dynamic systems based on piecewise Lyapunov functions," IEEE Trans. Fuzzy Syst., vol. 12, no. 1, pp. 22-28, Feb. 2004

[6] H. Gao, J. Lam, C. Wang, and Y. Wang, "Delay-dependent outputfeedback stabilisation of discrete-time systems with time-varying state delay," Proc. Inst. Elect. Eng.-Part D: Control Theory Appl., vol. 151, no. 6, pp. 691-698, Nov. 2004.

[7] H. Gao and C. Wang, "Comments and further results on 'A descriptor system approach to $H_{\infty}$ control of linear time-delay systems'," IEEE Trans. Autom. Control, vol. 48, no. 3, pp. 520-525, Mar. 2003.

[8] T. M. Guerra and L. Vermeiren, "LMI-based relaxed nonquadratic stabilization conditions for nonlinear systems in the Takagi-Sugeno's form," Automatica, vol. 40, no. 5, pp. 823-829, May 2004.

[9] Y. He, M. Wu, J. H. She, and G. P. Liu, "Delay-dependent robust stability criteria for uncertain neutral systems with mixed delays," Syst. Control Lett., vol. 51, no. 1, pp. 57-65, Jan. 2004.

[10] Y. He, M. Wu, J. H. She, and G. P. Liu, "Parameter-dependent Lyapunov functional for stability of time-delay systems with polytopictype uncertainties," IEEE Trans. Autom. Control, vol. 49, no. 5, pp. 828832, May 2004

[11] V. B. Kolmanovskii and J. P. Richard, "Stability of some linear systems with delays," IEEE Trans. Autom. Control, vol. 44, no. 5, pp. 984-989, May 1999. 
[12] C. H. Lien, "Further results on delay-dependent robust stability of uncertain fuzzy systems with time-varying delay," Chaos Solitons Fractals, vol. 28, no. 2, pp. 422-427, Apr. 2006.

[13] C. Lin, Q. G. Wang, and T. H. Lee, "Improvement on observer-based $H_{\infty}$ control for T-S fuzzy systems," Automatica, vol. 41, no. 9, pp. 16511656, Sep. 2005.

[14] C. Lin, Q. G. Wang, and T. H. Lee, "Stabilization of uncertain fuzzy timedelay systems via variable structure control approach," IEEE Trans. Fuzzy Syst., vol. 13, no. 6, pp. 787-798, Dec. 2005.

[15] H. Liu, F. Sun, and Y. N. Hu, " $H_{\infty}$ control for fuzzy singularly perturbed systems," Fuzzy Sets Syst., vol. 155, no. 2, pp. 272-291, Oct. 2005.

[16] H. Liu, F. Sun, and Z. Q. Sun, "Stability analysis and synthesis of fuzzy singularly perturbed systems," IEEE Trans. Fuzzy Syst., vol. 13, no. 2, pp. 273-284, Apr. 2005.

[17] X. Liu, "Delay-dependent $H_{\infty}$ control for uncertain fuzzy systems with time-varying delays," J. Comput. Appl. Math., vol. 68, no. 5, pp. 1352 $1361,2008$.

[18] Y. Liu, Z. Wang, and X. Liu, "Robust $H_{\infty}$ filtering for discrete nonlinear stochastic systems with time-varying delay," J. Comput. Appl. Math., vol. 341, no. 1, pp. 318-336, 2008.

[19] Y. Liu, Z. Wang, and X. Liu, "Robust stability of discrete-time stochastic neural networks with time-varying delays," Neurocomputing, vol. 71, no. 4-6, pp. 823-833, Jan. 2008.

[20] Y. S. Moon, P. Park, W. H. Kwon, and Y. S. Lee, "Delay-dependent robust stabilization of uncertain state-delayed systems," Int. J. Control, vol. 74, no. 14 , pp. $1447-1455$, Sep. 2001.

[21] T. Mori, N. Fukuma, and M. Kuwahara, "Delay-independent stability criteria for discrete-delay systems," IEEE Trans. Autom. Control, vol. AC-27, no. 4, pp. 964-966, Aug. 1982.

[22] L. A. Mozelli, C. D. Campos, R. M. Palhares, L. A. B. Tórres, and E. M. A. M. Mendes, "Chaotic synchronization and information transmission experiments: A fuzzy relaxed $H_{\infty}$ control approach," Circuits Syst. Signal Process., vol. 26, no. 4, pp. 427-449, Aug. 2007.

[23] S. K. Nguang and P. Shi, " $H_{\infty}$ fuzzy output feedback control design for nonlinear systems: An LMI approach," IEEE Trans. Fuzzy Syst., vol. 11, no. 3, pp. 331-340, Jun. 2003.

[24] P. Shi, E. K. Boukas, Y. Shi, and R. K. Agarwal, "Optimal guaranteed cost control of uncertain discrete time-delay systems," J. Comput. Appl. Math., vol. 157, no. 2, pp. 435-451, Aug. 2003.

[25] P. Shi and S. K. Nguang, " $H_{\infty}$ output feedback control of fuzzy system models under sampled measurements," Comput. Math. Appl., vol. 46, no. 5/6, pp. 705-717, 2003.

[26] T. Takagi and M. Sugeno, "Fuzzy identification of systems and its applications to modeling and control," IEEE Trans. Syst., Man, Cybern., vol. SMC-15, no. 1, pp. 116-132, Jan. 1985.

[27] K. Tanaka, A Theory of Advanced Fuzzy Control. Tokyo, Japan: Kyouritsu, 1994.

[28] K. Tanaka, T. Hori, and H. O. Wang, "A multiple Lyapunov function approach to stabilization of fuzzy control systems," IEEE Trans. Fuzzy Syst., vol. 11, no. 4, pp. 582-589, Aug. 2003.

[29] K. Tanaka and H. O. Wang, Fuzzy Control Systems Design and Analysis: A Linear Matrix Inequality Approach. New York: Wiley, 2001.

[30] Z. Wang, D. W. C. Ho, and X. Liu, "A note on the robust stability of uncertain stochastic fuzzy systems with time-delays," IEEE Trans. Syst., Man, Cybern. A, Syst., Humans, vol. 34, no. 4, pp. 570-576, Jul. 2004.

[31] Z. Wang, J. Lam, and K. J. Burnham, "Stability analysis and observer design for neutral delay systems," IEEE Trans. Autom. Control, vol. 47, no. 3, pp. 478-483, Mar. 2002.

[32] H. Wu, "Reliable LQ fuzzy control for nonlinear discrete-time systems via LMIs," IEEE Trans. Syst., Man, Cybern. B, Cybern., vol. 34, no. 2, pp. 1270-1275, Apr. 2004.

[33] H. Wu, "Delay-dependent stability analysis and stabilization for discretetime fuzzy systems with state delay: A fuzzy Lyapunov-Krasovskii functional approach," IEEE Trans. Syst., Man, Cybern. B, Cybern., vol. 36, no. 4, pp. 954-962, Aug. 2006.

[34] S. Xu and J. Lam, "Improved delay-dependent stability criteria for timedelay systems," IEEE Trans. Autom. Control, vol. 50, no. 3, pp. 384-387, Mar. 2005

[35] L. A. Zadeh, "Fuzzy sets," Inf. Control, vol. 8, pp. 338-353, 1965.

[36] S. Zhou, G. Feng, J. Lam, and S. Xu, "Robust $H_{\infty}$ control for discretetime fuzzy systems via basis-dependent Lyapunov functions," Inf. Sci., vol. 174, no. 3/4, pp. 197-217, Aug. 2005.

[37] S. Zhou and T. Li, "Robust stabilization for delayed discrete-time fuzzy systems via basis-dependent Lyapunov-Krasovskii function," Fuzzy Sets Syst., vol. 151, no. 1, pp. 139-153, Apr. 2005.

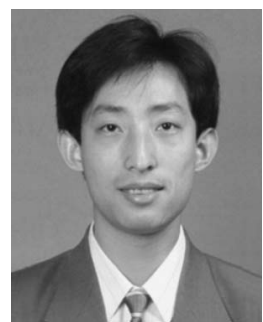

Huijun Gao (M'06) was born in Heilongjiang, China, in 1976. He received the M.S. degree in electrical engineering from the Shenyang University of Technology, Shengyang, China, in 2001 and the $\mathrm{Ph} . \mathrm{D}$. degree in control science and engineering from the Harbin Institute of Technology, Harbin, China, in 2005 .

He was a Research Associate with the Department of Mechanical Engineering, The University of Hong Kong, Pok Fu Lam, Hong Kong, from November 2003 to August 2004. From October 2005 to September 2007, he carried out his postdoctoral research with the Department of Electrical and Computer Engineering, University of Alberta, Edmonton, AB, Canada, supported by an Alberta Ingenuity Fellowship and an Honorary Izaak Walton Killam Memorial Postdoctoral Fellowship. Since November 2004, he has been with the Harbin Institute of Technology, where he is currently a Professor with the Department of Control Science and Engineering. He is an Associate Editor of the Journal of Intelligent and Robotics Systems, the International Journal of Innovative Computing, and Information and Control. He serves on the Editorial Board of the International Journal of Systems Science, the Journal of the Franklin Institute, and Nonlinear Dynamics and Systems Theory. His research interests include network-based control, robust control/filter theory, model reduction, time-delay systems, multidimensional systems, and their engineering applications.

Dr. Gao is an Associate Editor of the IEEE TRANSACTIONS ON SYSTEMS, MAN, AND CYBERNETICS-PART B: CYBERNETICS. He was the recipient of the University of Alberta Dorothy J. Killam Memorial Postdoctoral Fellow Prize in 2005 and a corecipient of the Outstanding Science and Technology Development Awards from the Ministry of Machine-Building Industry of China and from the Liaoning Provincial Government of China, both in 2002.

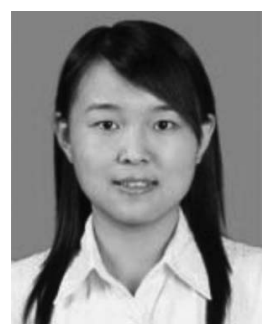

Xiuming Liu received the B.S. degree in information and computational science from the Harbin Engineering University, Harbin, China, in 2007. She is currently working toward the M.S. degree in the Department of Control Science and Engineering, Harbin Institute of Technology, Harbin.

Her research interests include fuzzy control systems and time-delay systems.

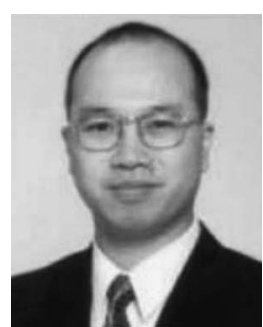

James Lam (S'86-M'86-SM'99) received the firstclass B.Sc. degree in mechanical engineering from the University of Manchester, Manchester, U.K., and the M.Phil. and Ph.D. degrees in control engineering from the University of Cambridge, Cambridge, U.K.

$\mathrm{He}$ is currently a Professor with the Department of Mechanical Engineering, The University of Hong Kong, Pok Fu Lam, Hong Kong. Prior to that, he held faculty positions at the City University of Hong Kong, Kowloon, Hong Kong, and the University of Melbourne, Melbourne, Australia. He is holding guest professorships with the Huazhong University of Science and Technology, Wuhan, China; South China University of Technology, Guangzhou, China; Shandong University, Shandong, China; and Central South University, Changsha, China. His research interests include reduced-order modeling, delay systems, descriptor systems, stochastic systems, multidimensional systems, robust control, and filtering. He has published numerous research articles in these areas and coauthored a monograph titled Robust Control and Filtering of Singular Systems.

Dr. Lam is an Associate Editor for the Asian Journal of Control, the International Journal of Systems Science, the Journal of Sound and Vibration, the International Journal of Applied Mathematics and Computer Science, the IEEE TRANSACTIONS ON Signal Processing, the Journal of the Franklin Institute, and the Conference Editorial Board of the IEEE Control Systems Society. $\mathrm{He}$ is also an editorial member of the IET Control Theory and Applications and the Open Electrical and Electronic Engineering Journal. He has served as the Editor-in-Chief of the IEE Proceedings: Control Theory and Applications and the IFAC Technical Committee on Control Design. He is a Scholar and a Fellow of the Croucher Foundation. He is a Chartered Mathematician and a Chartered Scientist, a Fellow of the Institute of Mathematics and Its Applications, and a member of the IEE. He was the recipient of the Ashbury Scholarship, the A.H. Gibson Prize, and the H. Wright Baker Prize for his academic performance. 